\title{
Lattice String Breaking and Heavy Meson Decays
}

\author{
I T Drummond and R R Horgan \\ Department of Applied Mathematics and Theoretical Physics \\ University of Cambridge \\ Silver St \\ Cambridge, England CB3 9EW
}

January 29, 2018

\begin{abstract}
We show how string breaking on the lattice, treated as a mixing effect, can be related to decay rates for heavy quark systems. We use this to make a preliminary calculation of the energy split at maximum mixing for static quarks in QCD from the decay rate for $\Upsilon(4 S) \rightarrow B \bar{B}$. We extend the calculation to achieve rough estimates for the contributions of channels involving $B, B^{*}, B_{s}$ and $B_{s}^{*}$ mesons to the width of the $\Upsilon(5 S)$.
\end{abstract}

DAMTP-98-127 


\section{Introduction}

It has been recognised for a long time that, just as the confining string-like interquark potential provides a good basis for understanding valence quark dynamics, so the breaking of the string provides a natural way of understanding the influence of sea quarks on decay processes [1, 2]. Lattice measurements of the confining QCD potential, in quenched and unquenched calculations, are well understood [3, 4, 5, 6, 7, 8, 9]. Moreover the simplest type of quark model using this potential gives a reasonable account of

the observed heavy meson spectrum. Recently there has been progress in the attempt to observe string breaking effects directly on the lattice [10, 11, 12].

In this paper we investigate how sufficiently accurate lattice measurements of the interquark potential and string breaking effects might be combined to implement a programme of computation of decay rates for heavy quark systems. The analysis depends on a two-channel mixing picture of the string breaking process suggested by a crude model proposed previously [13, 14, 15]. The basis of the mechanism is the fact that the light quark-anti-quark pair is produced (or absorbed) in a triplet spin state. Although our picture of string breaking is different in detail from that of Isgur and Paton [1] the results are still consistent with the phenomenological ${ }^{3} P_{0}$-model .

\section{Crude Model}

The crude model described previously [13, 14, 15], was designed to provide a simple two-channel mixing model of string breaking. It suggests that when the string breaks each light quark and anti-quark materialises in the neighbourhood of the appropriate static anti-quark or quark. In this respect it differs from the string breaking model of Isgur and Paton [1] who viewed the materialisation of the light quark-anti-quark pair as a local event on the string. As a justification of our picture we point out that to a first approximation the local gauge field energy density does not change as the static quarks are separated. There is no real mechanism therefore for the local production of the light pair. The breaking event is only possible when the total energy distributed along the string is sufficient to support light pair creation. Our model then suggests that this creation occurs essentially instantaneously in such a way that the gauge field distribution supported by the static quarks is cancelled by the opposing gauge field structure of the light quarks. It is this idea of instantaneous transition that we exploit below in our development of the model. However the outcome of the these 
considerations is not in contradiction with the ${ }^{3} P_{0}$ model that is supported by the analysis of Isgur and Paton, at least for the decay processes to which we apply our ideas.

As was pointed out in the original description of the crude model [13, 14, 15], the dynamical quark-anti-quark pair are produced in a correlated spin state. Here we examine this mechanism a little more closely. In the model the strong coupling graph that corresponds to the production of the pair is shown in Fig 1 . The quark or antiquark binds in an $S$-wave with the appropriate static anti-quark or quark. Choosing 0 as the (imaginary) time direction and 1 as the direction of spatial separation of the static quarks, we see that the rules for quark propagation [13, 14, 15, 19, 20] yield an amplitude with spin structure of the form

$$
\frac{1+\gamma_{0}}{2} \frac{1+\gamma_{1}}{2} \frac{1-\gamma_{0}}{2}=\frac{1}{2}\left(\begin{array}{cc}
0 & \sigma_{1} \\
0 & 0
\end{array}\right) .
$$

This can be interpreted as meaning that the light quark-anti-quark pair are produced with a spin wave function

$$
\xi_{\alpha \beta}=\frac{1}{\sqrt{2}}\left(\sigma_{i} \hat{\mathbf{R}}_{i}\right)_{\alpha \beta}
$$

where $\alpha$ and $\beta$ are the spin labels for the quark and anti-quark respectively and $\hat{\mathbf{R}}$ is the unit vector pointing along the line of separation of the the static quarks. This shows that the light quark-anti-quark pair are produced in a triplet state with zero spin component along the line of separation of the static quarks.

\section{Basic Mixing Scenario}

In order to explain the basic idea we first consider a model in which the particles of the theory have no spin but do experience confinement. The non-abelian Higgs models are of this type [10, 11]. Moreover they exhibit string breaking on the lattice of a type consistent with a two-channel mixing scenario [13, 14, 15]. The channels involved are

1) a static quark-anti-quark $\{Q \bar{Q}\}$ system with a separation $R$ and with a connecting flux string

2) a heavy-light meson-meson system $\{Q \bar{q}, q \bar{Q}\}$.

For the moment, we ignore the spins of the quarks and treat them as scalar particles. 
On the lattice it is possible to measure the string-string correlator $\mathcal{G}_{S S}(T)$ as the $R \times T$ Wilson loop. It has the form

$$
\mathcal{G}_{S S}(T) \sim e^{-V_{Q \bar{Q}}(R) T}
$$

where $V_{Q \bar{Q}}(R)$ can be interpreted as the interquark potential experienced by the static quarks. Lattice measurents are consistent with the Cornell potential [16, 17, 18]

$$
V_{Q \bar{Q}}(R)=C-\frac{\alpha}{R}+\sigma R
$$

where $\sigma$ is the string tension. The correlator for two-meson states over the time interval $T$ can be obtained from measurements of observables appropriate to the two-meson state. Its behaviour is of the form

$$
\mathcal{G}_{M M}(T) \sim e^{-E_{M M}(R) T}
$$

where $E_{M M}(R)=2 E_{M}+V_{M M}(R)$ and $E_{M}$ is the energy of a single static meson and $V_{M M}(R)$ is the potential interaction between the two static mesons. This potential energy has not been measured in QCD but does show up in the Higgs model calculations. We might expect it to have a form like [15

$$
V_{M M}(R)=W_{M} e^{-m R}
$$

where $m$ is the mass of a light meson that can be exchanged between the heavy mesons and $W_{M}$ is an energy determining the overall strength of the interaction.

This description is of course an oversimplification in the presence of dynamical matter fields. This is clear from the Higgs model calculations [10, 11] and in QCD from the thermal Polyakov loop calculations [12]. The reason is that string breaking can occur as a process that leads to a mixing of the string and two-meson states. Consistently with these static model calculations we may represent the mixing process by a transition potential $V_{I}(R)$. On the basis of the crude model we might expect it to have the form 13, 14, 15,

$$
V_{I}(R)=W e^{-m_{q} R}
$$

where $m_{q}$ is the mass of the dynamical matter field and $W$ is the energy parameter determining the strength of the transition potential. It follows that we are dealing with a two-channel interaction matrix of the form

$$
V(R)=\left(\begin{array}{cc}
V_{Q \bar{Q}}(R) & V_{I}(R) \\
V_{I}(R) & E_{M M}(R)
\end{array}\right)
$$


The energies measured directly on the lattice are the eigenvalues of $V(R)$. They are

$$
V_{ \pm}(R)=\frac{1}{2}\left\{V_{Q \bar{Q}}(R)+E_{M M}(R) \mp \sqrt{\left(V_{Q \bar{Q}}(R)-E_{M M}(R)\right)^{2}+4\left(V_{I}(R)\right)^{2}}\right\} .
$$

We keep the convention used previously that $V_{+}(R)$ is the lower of the two eigenenergies. If $V_{I}(R)$ is sufficiently small then the eigenenergies are dominated by the diagonal elements of $V(R)$. We can define a critical value of $R_{c}$ for which these diagonal values are equal

$$
V_{Q \bar{Q}}\left(R_{c}\right)=E_{M M}\left(R_{c}\right)
$$

When $R=R_{c}$ the the split in the eigenenergies is proportional to $V_{I}\left(R_{c}\right)$, we have

$$
V_{ \pm}\left(R_{c}\right)=V_{Q \bar{Q}}\left(R_{c}\right) \mp V_{I}\left(R_{c}\right)
$$

For $R<<R_{c}$ we expect $V_{Q \bar{Q}}(R)<<E_{M M}(R)$ with the result $V_{+}(R) \simeq V_{Q \bar{Q}}(R)$ and $V_{-}(R) \simeq E_{M M}(R)$. For $R>>R_{c}$ we expect $V_{Q \bar{Q}}(R)>>E_{M M}(R)$ with the result $V_{-}(R) \simeq V_{Q \bar{Q}}(R)$ and $V_{+}(R) \simeq E_{M M}(R)$. The interchange that occurs near $R=R_{c}$ is the mixing phenomenon. Just as in refs [13, 14, 15] we can compute a mixing angle $\theta$ given by

$$
\tan \theta=\frac{-\left(V_{Q \bar{Q}}(R)-E_{M M}(R)\right)+\sqrt{\left(V_{Q \bar{Q}}(R)-E_{M M}(R)\right)^{2}+4\left(V_{I}(R)\right)^{2}}}{2 V_{I}(R)} .
$$

We have

$$
V(R)=O\left(\begin{array}{cc}
V_{+}(R) & 0 \\
0 & V_{-}(R)
\end{array}\right) O^{-1}
$$

where

$$
O=\left(\begin{array}{cc}
\cos \theta & -\sin \theta \\
\sin \theta & \cos \theta
\end{array}\right)
$$

As $R$ passes from below to above $R_{c}$, the mixing angle increases from 0 to $\pi / 2$ hitting $\pi / 4$ when $R=R_{c}$. The goal then is to use lattice measurements to determine the eigenenergies and the $R$-dependence of the mixing angle. By those means we can determine the full two-channel interaction matrix, $V(R)$. We discuss this in more detail below.

Once we know the elements of $V(R)$ we can make the two-channel equivalent of the Born-Oppenheimer approximation and replace the static quarks by heavy nonrelativistic quarks and mesons. The effective Schrödinger equation is

$$
i \frac{\partial}{\partial t} \psi(\mathbf{R})=\left(\begin{array}{cc}
-\frac{1}{2 \mu_{Q}} \nabla^{2} & 0 \\
0 & -\frac{1}{2 \mu_{M}} \nabla^{2}
\end{array}\right) \psi(\mathbf{R})+V(R) \psi(\mathbf{R})
$$


where

$$
\psi(\mathbf{R})=\left(\begin{array}{c}
\psi_{Q}(\mathbf{R}) \\
\psi_{M}(\mathbf{R})
\end{array}\right)
$$

and $\mu_{Q}$ and $\mu_{M}$ are the reduced masses for the heavy quarks and heavy mesons in their respective channels. These also can be obtained from appropriate lattice measurements of heavy single meson states.

We can exploit eq(15) by first ignoring the off-diagonal elements of $V(R)$. We then calculate the heavy $Q \bar{Q}$ bound states with energies $E_{Q \bar{Q}}$. If this energy lies above the two-meson channel threshold then we can compute the decay rate as

$$
\Gamma=2 \pi \rho(k)|T(k)|^{2}
$$

where $E_{Q \bar{Q}}$ is the energy of the bound state $Q \bar{Q}$ system in the potential $V_{Q \bar{Q}}(R), \mathbf{k}$ is the relative momentum of the final state mesons, $\rho(k)$ is the density of states factor. The energy of the two-meson state is $E(k)$ which satisfies

$$
E(k)=\frac{1}{2 \mu_{M}} k^{2}+2 E_{M}=E_{Q \bar{Q}}
$$

The transition amplitude is

$$
T(k)=\left\langle\mathbf{k}\left|V_{I}\right| \psi_{Q}\right\rangle
$$

$|\mathbf{k}\rangle$ being the wavefunction, with incoming scattered wave, for the two meson system in the presence of the potential $V_{M M}(R)$. As a first approximation we could neglect the the final state interactions represented by the scattered part of the the wave function and replace $|\mathbf{k}\rangle$ with the plane wave $e^{i \mathbf{k} . \mathbf{R}}$. We have then

$$
T(k)=\int d^{3} \mathbf{R} e^{-i \mathbf{k} \cdot \mathbf{R}} V_{I}(R) \psi_{Q}(R)=4 \pi \int_{0}^{\infty} d R R^{2}\left(\frac{\sin k R}{k R}\right) V_{I}(R) \psi_{Q}(R)
$$

In the above it is assumed that the static approximation for the mesons is accurate in the sense that

$$
E_{Q \bar{Q}}-2 E_{M}=M_{Q \bar{Q}}-2 M_{M}
$$

where $M_{Q \bar{Q}}$ and $M_{M}$ are the actual masses of the heavy quark state and the heavy-light meson state respectively. To the extent that the results are only approximate the left side of eq(21) should be replaced by the right side in an actual estimate of the decay rate. 


\section{Lattice Measurement of the Interaction Matrix}

On the lattice we can measure $V_{ \pm}(R)$ [10, 11, [12]. If we are confident of our parametrizations of the elements of $V(R)$, then it is likely that we can determine them by performing a fit to the eigenenergies provided they are known sufficiently accurately and on a sufficiently fine spatial lattice.

In principle, the the eigenenergies are not sufficient to fix the elements of $V(R)$. A superior approach, therefore, would be to measure also the mixing angle $\theta$. The results obtained in the $S U(2)$-Higgs models for the $R$-dependence of the ground state overlap of the lower eigenmode suggest that this may well be possible [10, 11]. The two-channel model suggests that the contribution of the lower energy eigenmode to the Wilson loop is

$$
\mathcal{G}_{S S}(R) \simeq \Gamma \cos \theta e^{-V_{+}(R) T} \Gamma \cos \theta,
$$

where $\Gamma$ is independent of $R$. If we make this assumption then then we can deduce the $R$-dependence of $\theta$ from the measurement of $\mathcal{G}_{S S}(R)$.

It would be interesting if this scenario could be generalized to a set of observables $A_{i}(R, T) i=1, \ldots, N$ for which the correlators have the form

$$
\left\langle A_{i}(R, T) A_{j}(R, 0)\right\rangle=\sum_{\alpha= \pm} \Gamma_{i \alpha}(R) e^{-V_{\alpha}(R) T} \Gamma_{j \alpha}(R),
$$

where the $R$-dependence of the coupling coefficients comes from the movement of the mixing angle. That is

$$
\Gamma_{i \alpha}(R)=\sum_{a=S, M} \Gamma_{i a} O_{a \alpha}(R) .
$$

Introducing the $N$-component vectors $\mathbf{C}_{\alpha}=\left\{\Gamma_{i \alpha}\right\}$ and $\mathbf{C}_{a}=\left\{\Gamma_{i a}\right\}$ we easily see from the the form of the orthogonal matrix $O$ given in eq(14) that

$$
\mathbf{C}_{+}(R) \wedge \mathbf{C}_{-}(R)=\mathbf{C}_{S} \wedge \mathbf{C}_{M}
$$

and

$$
\mathbf{C}_{i+}(R)^{2}+\mathbf{C}_{i-}(R)^{2}=\mathbf{C}_{i S}^{2}+\mathbf{C}_{i M}^{2} \quad i=1, \ldots, N .
$$

If the left sides of these equations do show a lack of dependence on $R$ then we would have a good test of the the above coupling hypothesis. It is not obvious that all sets of operators have this property but it may be possible to construct a sufficient set. However we might reasonably expect in general that the $R$-dependence of these 
quantities is relatively weak compared to that induced by the rapid variation of the mixing angle through the mixing region.

On the assumption that an acceptable set of operators can be found, the dependence of $\theta$ on $R$ can be elucidated by examining the quantities $y_{+}=\mathbf{n} \cdot \mathbf{C}_{+}(R)$ and $y_{-}=\mathbf{n} . \mathbf{C}_{-}(R)$ where $\mathbf{n}$ is any appropriate $N$-vector. They have the form

$$
\left(\begin{array}{l}
y_{+} \\
y_{-}
\end{array}\right)=\left(\begin{array}{cc}
\cos \theta & -\sin \theta \\
\sin \theta & \cos \theta
\end{array}\right)\left(\begin{array}{c}
y_{S} \\
y_{M}
\end{array}\right),
$$

where $y_{S}=\mathbf{n} \cdot \mathbf{C}_{S}$ and $y_{M}=\mathbf{n} \cdot \mathbf{C}_{M}$. Clearly the point $\left(y_{+}, y_{-}\right)$lies on a circle and $\theta$ is the angle, referred to an appropriate origin, that fixes the position of the point on the circle.

\section{$5 \quad$ Light Quarks with Spin}

Static quark calculations are particularly relevant to the spectator quark approximation in which, except for determining multiplicities, the heavy quark spins play essentially no rôle. Of course in the physical case of bottomonium, the small mass differences associated with the hyper-fine structure of the $B$-mesons are crucial in determining allowed decays. These effects are however essentially kinematic and will be taken into account at the appropriate point. In examining the basic phenomenon of string breaking we will temporarily omit these spins from consideration. The light quark spins however play a crucial part in both string breaking and decay dynamics.

As discussed in section 2 our crude model provides a generalization of the scalar case to that of light quarks with spin. It remains a two-channel mixing problem because the light quark-anti-quark pair emerges in a triplet spin state with definite orientation. We can achieve this result by introducing a transition potential of the form

$$
V(\mathbf{R})=\left(\begin{array}{cc}
V_{Q \bar{Q}}(R) & V_{M S}(\mathbf{R}) \\
V_{M S}^{\dagger}(\mathbf{R}) & E_{M M}(R)
\end{array}\right),
$$

where now the spin label structure of the transition element is given by

$$
V_{M S \alpha \beta}(\mathbf{R})=\frac{1}{\sqrt{2}} V_{I}(R)\left(\sigma_{i} \hat{\mathbf{R}}_{i}\right)_{\alpha \beta}
$$

This indeed implies that $V_{M S}(\mathbf{R})$ couples the static quark-anti-quark state to a two-

meson state in which the light quark spins are in a state represented by the wave 
function $\xi_{\alpha \beta}$ given in eq(2) . Projecting down onto the appropriate subspace the two channel structure emerges and the effective potential matrix is

$$
V^{\mathrm{eff}}(R)=\left(\begin{array}{cc}
V_{Q \bar{Q}}(R) & V_{I}(R) \\
V_{I}(R) & E_{M M}(R)
\end{array}\right)
$$

This is of precisely the form of the interaction matrix in the scalar case and the eigenvalues $V_{ \pm}(R)$ which which are given by eq $(9)$ are the energies that will be computed on the lattice from the measurement of appropriate operators. In the same spirit as for the scalar calculation we can expect, through careful lattice measurements, to determine the entries in $V^{\mathrm{eff}}(R)$. Having done that we revert to considering the Schrödinger equation

$$
i \frac{\partial}{\partial t} \psi(\mathbf{R})=\left(\begin{array}{cc}
\frac{1}{2 \mu_{Q}} \nabla^{2} & 0 \\
0 & \frac{1}{2 \mu_{M}} \nabla^{2}
\end{array}\right) \psi(\mathbf{R})+V(\mathbf{R}) \psi(\mathbf{R}),
$$

Following the pattern set out for the scalar case we calculate the decay rate by solving eq(31) with $V_{I}(R)=0$ for an $S$-wave bound by $V_{Q \bar{Q}}(R)$ and a scattering wave controlled by $V_{M M}(R)$. The transition rate is then

$$
\Gamma=2 \pi \sum_{i} \rho(k)\left|T_{i}(\mathbf{k})\right|^{2}
$$

where

$$
T_{i}(\mathbf{k})=\left\langle\mathbf{k} i\left|V_{M S}(\mathbf{R})\right| \psi_{Q}\right\rangle,
$$

and $i$ is the polarization state of the triplet spin wavefunction. In principle the state $|\mathbf{k} i\rangle$ should be a solution of the the scattering problem with the potential $V_{M M}(R)$. If we make the approximation of neglecting final state interactions we can set

$$
|\mathbf{k} i\rangle=\frac{1}{\sqrt{2}} \sigma_{i} e^{i \mathbf{k} \cdot \mathbf{R}}
$$

We have then

$$
T_{i}(\mathbf{k})=\frac{1}{\sqrt{2}} \int d^{3} \mathbf{R} e^{-i \mathbf{k} \cdot \mathbf{R}} \operatorname{Tr}\left\{\sigma_{i} V_{M S}(\mathbf{R})\right\} \psi_{Q}(R)
$$

That is

$$
T_{i}(\mathbf{k})=4 \pi i k_{i} \int_{0}^{\infty} d R R\left(\frac{\cos k R}{k^{2}}-\frac{\sin k R}{k^{3} R}\right) V_{I}(R) \psi_{Q}(R) .
$$

Note that $T_{i}(\mathbf{k}) \sim O(k)$ for small $k$. This is the angular momentum barrier associated with the $P$-wave final state. 


\section{Heavy Meson Decay}

Currently there is no detailed information on string breaking in QCD at zero temperature although there is a strong indication of the mixing effect at finite temperature [12]. The latest measurement of the $Q \bar{Q}$ potential with dynamical quarks does not show an unequivocal mixing effect directly [8]. This is not inconsistent with the mixing scenario if the mixing range in $R$ and the split in eigenenergies at maximal mixing is sufficiently small [13, 14, 15]. It is therefore interesting to reverse the above argument and use the information on the decay of the $\Upsilon(4 S)$ to $B \bar{B}$ to estimate the energy split at maximum mixing that we should expect to see on the lattice. The theory also enables us to make predictions for other bottomonium decays.

The hyperfine structure of the heavy-heavy and heavy-light quark systems has a strong effect on the relative positions of masses and thresholds and hence on decay rates. In order to make use of experimental results therefore, it is necessary to reinstate the spins of the heavy quarks. To compute the decay rates of real processes, we must take into account the spin structure of the open final state channels in order to assign the correct momenta to them.

The heavy quarks in the $\Upsilon$ are in a triplet state and continue, as spectator quarks, in that state after decay. We can represent this state by a spin wave function $\sigma_{j \alpha^{\prime} \beta^{\prime}} / \sqrt{2}$, where $\alpha^{\prime}$ is the heavy quark label and $\beta^{\prime}$ is the heavy anti-quark label. In our model the light quarks are produced in a triplet state that can be represented by a wave function

$\sigma_{i \alpha \beta} / \sqrt{2}$. A sufficiently complete basis for the achievable final states is

$$
\psi_{i j}=\frac{1}{2} \sigma_{i \alpha \beta} \sigma_{j \alpha^{\prime} \beta^{\prime}}
$$

The transition matrix element we require is then

$$
\left\langle\psi_{i^{\prime} j^{\prime}} \mathbf{k}\left|V_{M S}\right| \psi_{Q}, j\right\rangle=\delta_{j j^{\prime}} T_{i^{\prime}}(\mathbf{k})
$$

where $\mathbf{k}$ is the relative momentum for the final state mesons and $T_{i^{\prime}}(\mathbf{k})$ is the amplitude as previously defined. The spin state $\psi_{i j}$ can be decomposed into a physical basis such as $B \bar{B}, B \bar{B}^{*}+B^{*} \bar{B}, B^{*} \bar{B}^{*}(S=2)$ and $B^{*} \bar{B}^{*}(S=0)$. The resulting decay rate is given by eq(32) modified by a probability factor $p$ appropriate to the channel under consideration.

$$
\Gamma=p 2 \pi \rho(k) \sum_{i}\left|T_{i}(\mathbf{k})\right|^{2},
$$

These probabilities, calculated from the appropriate overlap coefficients in the spin wavefunction recoupling scheme, for the physical channels are 


\begin{tabular}{|c|c|c|c|}
\hline$B \bar{B}$ & $B \bar{B}^{*}+B^{*} \bar{B}$ & $B^{*} \bar{B}^{*}(S=2)$ & $B^{*} \bar{B}^{*}(S=0)$ \\
\hline $1 / 12$ & $1 / 3$ & $5 / 9$ & $1 / 36$ \\
\hline
\end{tabular}

Introducing the radial wavefunction $\chi(R)=\sqrt{4 \pi} R \psi_{Q}(R)$ and using eq(罒) we can compute $T_{i}(\mathbf{k})$ from eq(36) as

$$
T_{i}(\mathbf{k})=\sqrt{4 \pi} i k_{i} A\left(k, m_{q}\right) W,
$$

where

$$
A\left(k, m_{q}\right)=\int_{0}^{\infty} d R\left(\frac{\cos k R}{k^{2}}-\frac{\sin k R}{k^{3} R}\right) e^{-m_{q} R} \chi(R) .
$$

Finally the decay rate to a particular channel is

$$
\Gamma=p N_{f} 4 \mu k^{3}\left|A\left(k, m_{q}\right)\right|^{2} W^{2},
$$

where we have used $\rho(k)=\mu k /\left(2 \pi^{2}\right), \mu$ is the reduced mass of the final state particles and $N_{f}$ is the number of degenerate $B \bar{B}$ channels which is identical to the number of degenerate light quarks. We use mass values from the Review of Particle Physics [21],

\begin{tabular}{|c|r|r|r|r|r|r|}
\hline Mass & $M_{B}$ & $M_{B^{*}}$ & $M_{B_{s}}$ & $M_{B_{s}^{*}}$ & $M_{\Upsilon(4 S)}$ & $M_{\Upsilon(5 S)}$ \\
\hline $\mathrm{GeV}$ & 5.279 & 5.325 & 5.369 & 5.416 & 10.580 & 10.860 \\
\hline
\end{tabular}

We choose as a representation of $V_{Q \bar{Q}}(R)$ its parametrization in eq(四). A choice of parameter values that accord reasonably well with lattice measurements and the $\Upsilon(S)$ excitation spectrum is $\alpha=0.52$ and $\sigma=(0.429)^{2} \mathrm{GeV}^{2}$, together with a heavy quark reduced mass $\mu_{Q}=2.1 \mathrm{GeV}$. The resulting radial wavefunctions $\chi(R)$ for $\Upsilon(4 S)$ and $\Upsilon(5 S)$ are shown in Fig 2. Both wavefunctions remain substantial over a range of $10 \mathrm{GeV}^{-1} \simeq 2 \mathrm{fm}$ with oscillations near the origin of a half wavelength $\sim 1-2 \mathrm{GeV}^{-1}$ before reaching broad maxima at $\sim 5 \& 7 \mathrm{GeV}^{-1}$.

As indicated above we ignore final state interactions, effectively setting $V_{M M}(R)=0$. Because our calculation is a preliminary one we will assume in what follows that $m_{q}=$ 
0 . This may be justified as a rough approximation by the low masses of dynamical quarks. A crucial factor in computing the decay rate is the amplitude $A\left(k, m_{q}\right)$. We exhibit this amplitude, for the case $m_{q}=0$, as a function of the final state momentum $k$ in Fig 3 for the $\Upsilon(4 S)$ and $\Upsilon(5 S)$. Note that in both cases it is small in magnitude for $k>0.5 \mathrm{GeV}$. We can expect therefore as a general rule that only decays with a sufficiently small final state momentum will contribute substantially to the width of the initial state.

In our model the only open channel for $\Upsilon(4 S)$-decay is $B \bar{B}$. Experimentally this process dominates. The relative momentum of the the $B \bar{B}$ mesons in the final state is $k=\sqrt{M_{B}\left(M_{4 S}-2 M_{B}\right)}=.3408 \mathrm{GeV}$. This yields a value for the amplitude $A(k, 0)=$ -10.4 . From eq(42) with $N_{f}=2$ and $p=1 / 12$ we find

$$
\Gamma(\Upsilon \rightarrow B \bar{B})=7.02 W^{2}
$$

The experimental result is $\Gamma(\Upsilon \rightarrow B \bar{B})=0.010(5) \mathrm{GeV}$. This yields an estimate $W=0.038(9)$ Gev. In turn we can estimate the eigenenergy split at maximum mixing as $\Delta E=2 \sqrt{N_{f}} W=0.11(3) \mathrm{GeV}$. We can crudely estimate the range in $R$ over which the mixing takes place as

$$
\Delta R=\frac{\Delta E}{V_{Q Q}^{\prime}\left(R_{c}\right)} \simeq \frac{\Delta E}{\sigma},
$$

with the result $\Delta R \simeq 0.6 \mathrm{GeV}^{-1} \simeq 0.12 \mathrm{fm}$. To resolve such a split requires a rather fine lattice. The small value of this split, coupled with the decoupling of the two-meson state outside the mixing region may be regarded as an explanation of why current measurements of the Wilson loop do not yet reveal string breaking in lattice QCD. We can extend the above analysis to higher excitations such as the $\Upsilon(5 S)$ which has a number of open decay channels. The results, assuming $W$ has the same value as before, are 


\begin{tabular}{|c|c|c|c|c|}
\hline Channel & $N_{f}$ & $\mathrm{k}(\mathrm{GeV})$ & $A(k, 0)$ & $\Gamma(\mathrm{MeV})$ \\
\hline$B \bar{B}$ & 2 & 1.26 & -0.68 & 2.0 \\
$B \bar{B}^{*}+B^{*} \bar{B}$ & 2 & 1.16 & -0.734 & 9.0 \\
$B^{*} \bar{B}^{*}$ & 2 & 1.06 & -0.430 & 4.0 \\
$B_{s} \bar{B}_{s}$ & 1 & 0.81 & 1.651 & 2.0 \\
$B_{s} \bar{B}_{s}^{*}+B_{s}^{*} \bar{B}_{s}$ & 1 & 0.636 & 1.1545 & 3.0 \\
$B_{s}^{*} \bar{B}_{s}^{*}$ & 1 & 0.389 & -7.33 & 29.0 \\
\hline
\end{tabular}

The total width is $\Gamma \simeq 50 \mathrm{MeV}$ which is to be compared with the the quoted experimental result $\Gamma=110(13) \mathrm{MeV}$. Given the crudity of the theoretical approach and the experimental difficulties this is not an unreasonable comparison.

\section{Conclusions}

We have examined the relevance of string breaking to the decay of heavy quark systems. The anaysis shows how string breaking on the lattice can be related to the decay rates for such processes as $\Upsilon(4 S) \rightarrow B \bar{B}$. The central idea is that the string breaking can be viewed as a two channel process. This is controlled by a $2 \times 2$ potential matrix of which the diagonal elements represent the interquark potential and the energy and interaction potential of two static mesons. The off-diagonal elements correspond to a transition potential between the two channels. Just as the interquark potential can be used in a dynamical calculation of heavy quark states so the transition potential can be used to compute the transition rate from the bound quark system to two freely moving heavy mesons. This is a standard relatively non-controversial quantum mechanics calculation. The main assumption behind it is that the Born-Oppenheimer approximation that underpins the heavy quark calculation holds good when the transition matrix elements are included. This is equivalent to assuming that there is no strong retardation effect in the transmission of the dynamical quark. If this quark mass is sufficiently low this assumption is not unreasonable.

At the moment there are no measurements in QCD that permit an accurate realization of the scheme. However, with some simplifying assumptions on the form of the elements of the potential matrix, it is possible to make a preliminary calculation of the decay rate 
of the $\Upsilon(4 S)$ in terms of the energy parameter $W$, yielding a value $\simeq 38 \mathrm{MeV}$. This gives rise to an eigenenergy split of $\sim 110 \mathrm{MeV}$. Although this suggests that the split may be hard to detect on the lattice the study of mixing remains an important goal because of its physical significance. Our analysis also strongly supports the desirability of measuring an appropriate suite of operators for the purpose of measuring the movement of the mixing angle [10, 11]. In fact, as indicated in [15, it would be very useful in estimating $V_{I}(R)$ to measure the transition amplitude for string to two mesons in the quenched appoximation [22].

Taking the above value of $W$ as a guide we are able to use the formalism to compute the partial widths for $\Upsilon(5 S)$-decay. We find that the dominant decay is through the $B_{s}^{*} \bar{B}_{s}^{*}$ channel. Our computed total width is roughly half the measured width. We feel that this result is not unreasonable given the preliminary character of the calculation and the limited information available from experiment on decay channels.

We conclude that with some improvement of lattice measurements it will be possible to confront string breaking with experimental results when these become available. 


\section{References}

[1] N Isgur and J Paton, Phys Rev D31 (1985) 2910

[2] P Geiger and N Isgur, Phys Rev D41 (1990) 1595

[3] UKQCD Collaboration, S P Booth et al, Phys Letts B275 (1992) 424

[4] G Bali and K Schilling, Phys Rev D47 (1993) 661

[5] G Bali and K Schilling, Phys Rev D55 (1997) 5309

[6] G Bali and K Schilling, Phys Rev D56 (1997) 2566

[7] CP-PACS Collaboration, S Aoki et al, Nucl Phys Proc Suppl 63 (1998) 221

[8] R Burkhalter, Physics report from the CP-PACS collaboration, Plenary talk at LATTICE'98.

[9] CP-PACS Collaboration: T Kaneko, "The static quark potential in full QCD", talk at LATTICE'98.

[10] O Philipsen and H Wittig, "String breaking in Non-Abelian Gauge Theories with Fundamental Matter Fields", hep-lat/9807020 and "The static potential beyond the screening length: a 3d gauge Higgs model", talk at LATTICE'98.

[11] F Knechtli and R Sommer, "String Breaking in $S U(2)$ Gauge Theory with Scalar matter Fields", hep-lat/9807022 and

[12] C De Tar, C McNeile, F Karsch and E Laerman, "String Breaking in Lattice QCD", talk at LATTICE'98

[13] I T Drummond, "Strong Coupling Model for String Breaking on the Lattice", hep-lat/9805012, Phys. Letts. B434 (1998) 92-98

[14] I T Drummond, "Strong Coupling Model for String Breaking on the Lattice", talk at LATTICE'98", hep-lat/9807038.

[15] I T Drummond, "Mixing Scenarios for Lattice String Breaking", hep-lat/9808014

[16] E Eichten and K Gottfried, Phys. Letts. 66B (1977) 286 
[17] E Eichten, K Gottfried, T Kinoshita, K D Lane and T M Yan, Phys. Rev. D 17 (1978) 3090

[18] E Eichten, K Gottfried, T Kinoshita, K D Lane and T M Yan, Phys. Rev. D 21 (1980) 203

[19] M Creutz, "Quarks and gluons and lattices", Cambridge Monographs on Mathematical Physics, Cambridge University Press, (1983)

[20] I Montvay and G Münster, "Quantum Fields on a Lattice", Cambridge Monographs on Mathematical Physics, Cambridge University Press, 1994

[21] Particle Data Group, Review of Particle Physics, The European Physical Journal C3 (1998)

[22] "String Breaking in Quenched QCD", talk at LATTICE'98, hep-lat/9809063 and hep-lat/9811012 


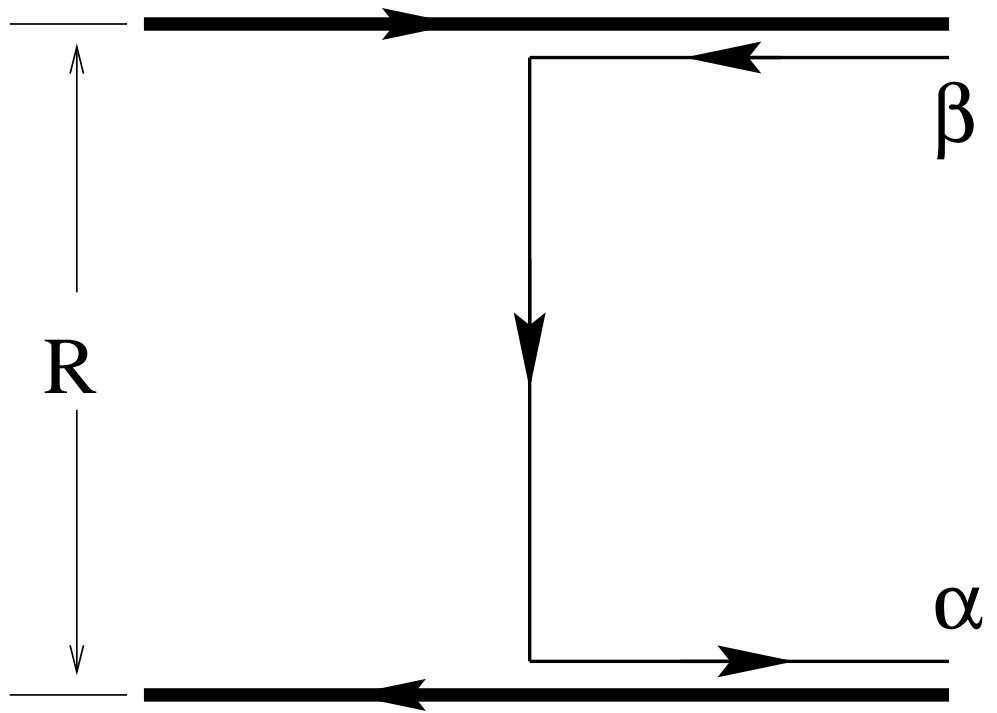

Figure 1: Transition from string to two-meson state. Heavy lines represent static quarks, light lines represent dynamical quarks. 


\section{Y: 4S (solid) 5S (dashed)}

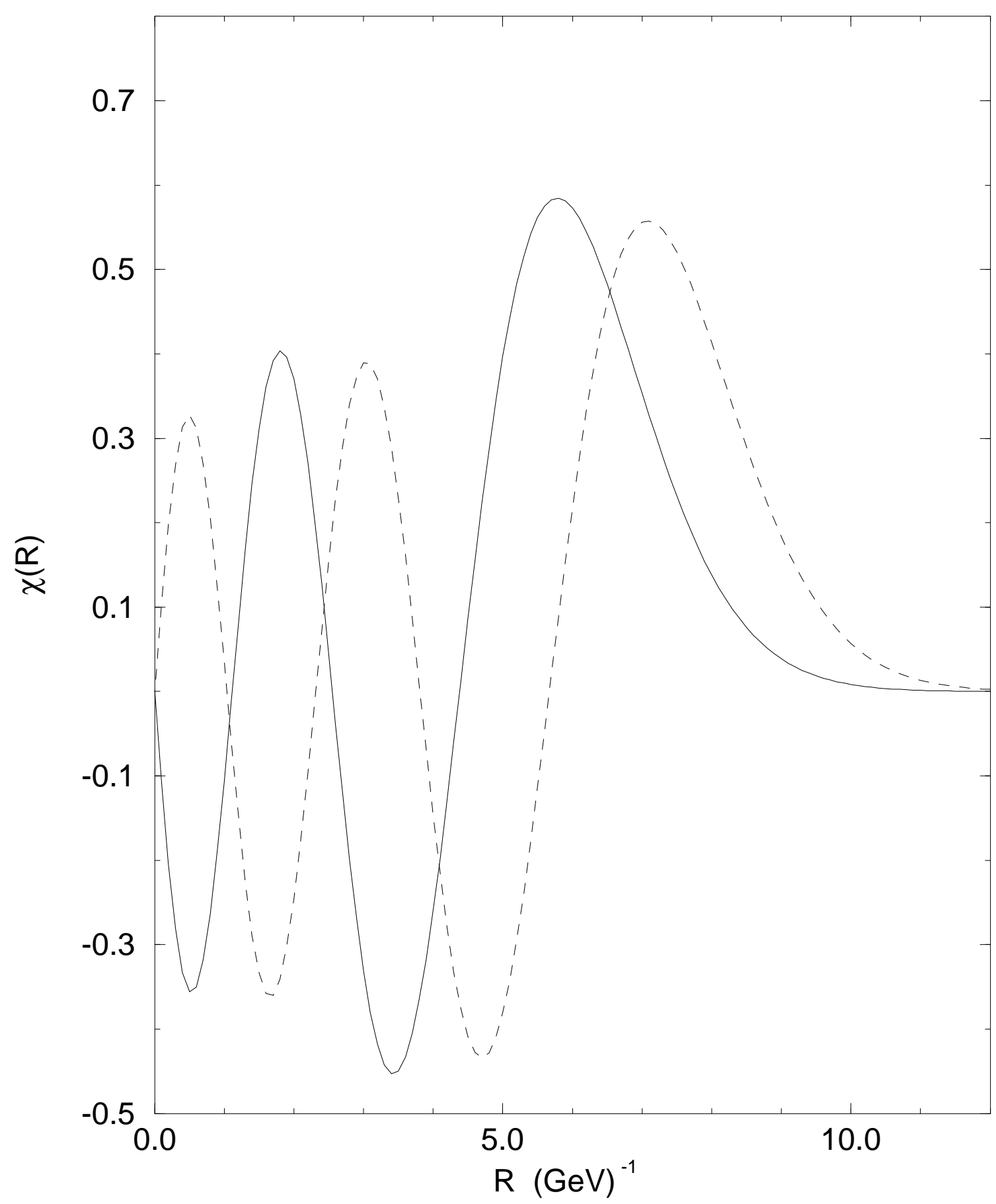

Figure 2: Wavefunctions for $\Upsilon(4 S)$ and $\Upsilon(5 S)$. 


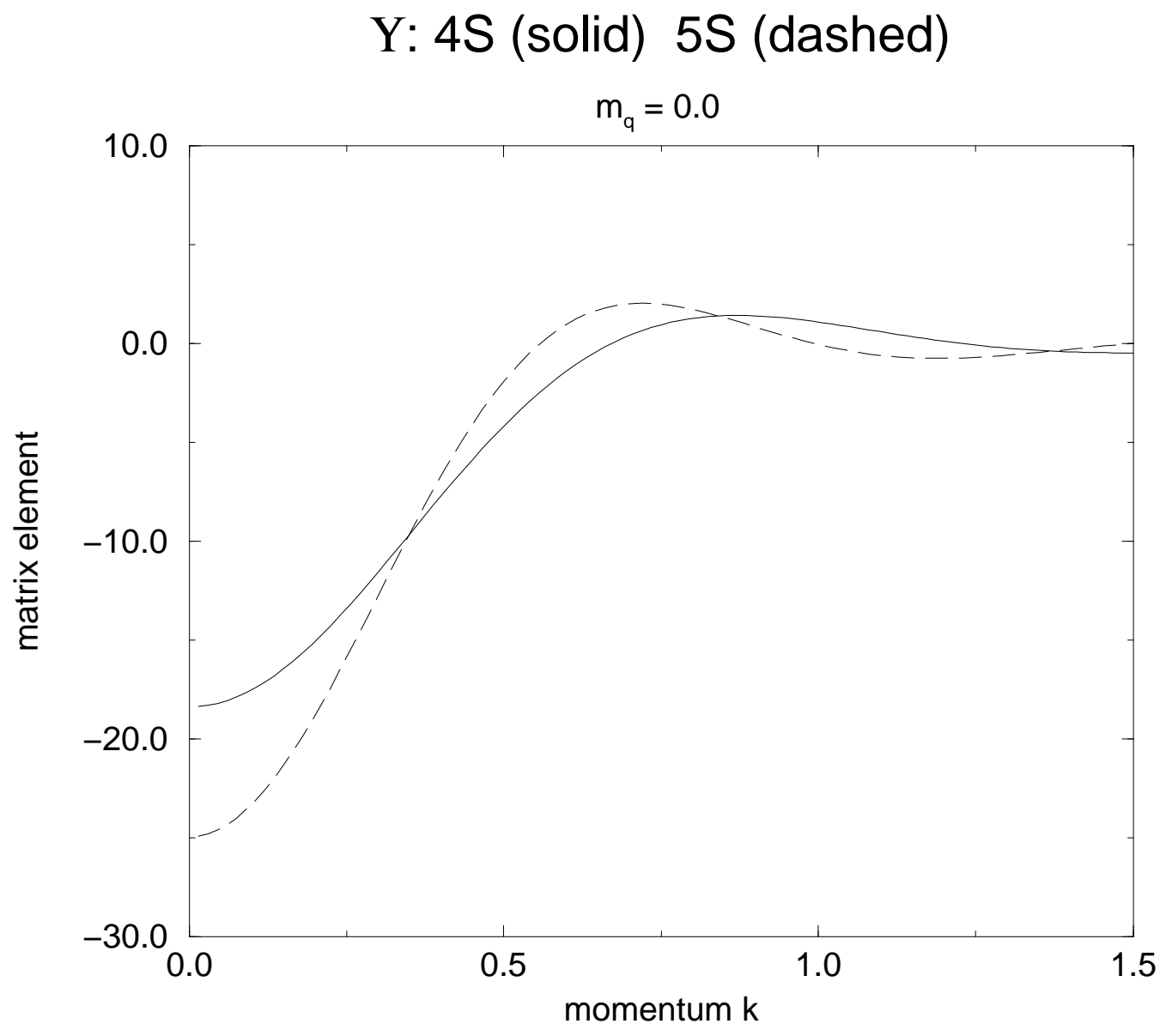

Figure 3: Transition matrix element, $A(k, 0)$, for $\Upsilon(4 S)$ and $\Upsilon(5 S)$. 